\title{
Polimorfismo de la proteína ligante de ácidos grasos intestinal (FABP2), obesidad e insulina resistencia
}

\author{
Cecilia Albala $B^{1}$, Beatriz Jiménez $R^{2}$, Francisco Pérez $B^{1 a}$, \\ Claudio Liberman $\mathbf{G}^{2}$. \\ Fatty acid binding protein 2 (FABP-2) \\ polymorphism, obesity \\ and insulin resistance
}

The genetic components of insulin-resistance, diabetes and obesity have been largely studied. These conditions are determined by multiple polygenic and environmental factors. Certain candidate genes, that have common functional variants in the general population, may be important determinants of inter-individual differences in the response to dietary changes. This review focuses in one of the major candidate genes, the gene encoding for the FABP2, an intracellular protein expressed only in the intestine, involved in the absorption and intracellular transport of dietary long chain fatty acids. Carriers of the Thr54 allele in FABP2 have a 2-fold greater affinity for long chain fatty acids than Ala54 carriers. The increased flux of dietary fatty acids (FA) into the circulation, among carriers of FABP2 Ala54Thr, supports a role of the polymorphism of this allele in the etiology of metabolic disorders. The frequencies of the polymorphism in different populations fluctuate between $18 \%$ and $40 \%$. FABP2 Ala54Thr variant has been associated with an increased fasting insulin concentration, fasting fatty acid oxidation and reduced glucose uptake. This evidence, although not conclusive, sustains an association between FABP-2 genotype and metabolic abnormalities (Rev Méd Chile 2006; 134: 372-9).

(Key words: Diabetes mellitus; FABP2 protein, human; Fatty acid-binding proteins; Obesity)

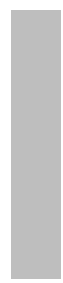

Recibido el 14 de marzo, 2005. Aceptado el 10 de agosto, 2005.

La investigación de FABP-2 en Chile ha sido financiada íntegramente por medio del Proyecto Fondecyt 1020703.

${ }^{1}$ Laboratorio de Investigación en Epidemiología Nutricional y Genética, Instituto de Nutrición y Tecnología de Alimentos (INTA), Universidad de Chile. ${ }^{2}$ Departamento de Endocrinología, Hospital Clínico de la Universidad de Chile y Facultad de Medicina, Universidad de Chile. Santiago de Chile.

${ }^{a}$ Doctor en Ciencias

$\overline{\text { Correspondencia a: Dra. Cecilia Albala Brevis. Laboratorio }}$ de Investigación en Epidemiología Nutricional y Genética, INTA, Universidad de Chile. Santiago - Chile. Casilla: 13811. E mail: calbala@uchile.cl 
$\mathrm{E}$ 1 componente genético de la resistencia insulínica (RI) ha sido ampliamente estudiado, existiendo evidencia sustancial de que las formas comunes de RI están fuertemente influidas por la herencia $^{1,2}$. Se ha observado que los parientes no diabéticos de sujetos diabéticos son más insulinoresistentes que los controles y que la variabilidad intrafamiliar de la sensibilidad insulínica es significativamente menor que su variabilidad interfamiliar ${ }^{3}$. La heredabilidad de la RI, es decir, la proporción de la variación fenotípica total atribuible al efecto aditivo de genes, se ha estimado a través de estudios de gemelos y de pedigríes en $60 \%$ para la RI y $35-54 \%$ para la insulinemia $1,3,4$. Tanto la RI, la diabetes tipo 2, como la obesidad son condiciones poligénicas y multifactoriales ${ }^{2}$. Dentro de los múltiples genes estudiados en relación con su expresión fenotípica, el polimorfismo de FABP2 es uno de los genes candidatos mayores asociados consistentemente con RI y obesidad $^{5}$.

Las FABP constituyen una familia de proteínas citoplasmáticas involucradas en el transporte y metabolismo intracelular de ácidos grasos (AG) de cadena larga 5 ,6 La familia de proteínas ligantes de AG está formada por más de veinte proteínas que han sido identificadas y numeradas de acuerdo a su tejido de expresión, incluyendo la FABP intestinal específica o FABP2. Su expresión es regulada por factores hormonales, factores de transcripción y factores ambientales como la composición de la $\operatorname{dieta}^{7}$.

\section{ESTRUCTURA Y FUNCIÓN}

La FABP2 participa en la transferencia y metabolismo intracelular de AG. El gen para la FABP2 se expresa solamente en las células del epitelio absortivo columnar simple del intestino delgado, donde la FABP2 transporta AG desde la membrana plasmática luminal hasta el retículo endoplásmico rugoso. Una vez allí, los AG son esterificados con el glicerol-3-fosfato para formar triglicéridos (TG), los que se almacenan dentro de los quilomicrones para ser transportados posteriormente por el plasma 5 .

La localización del gen que codifica la proteína FABP2 fue descrita por primera vez en 1987 por Sparkes y cols, en la región cromosomal 4q27-4q31 ${ }^{8}$.
La proteína FABP2 es una pequeña proteína citosólica compuesta de 131 aminoácidos con un peso molecular de $15 \mathrm{KDa}^{5}$. Su estructura terciaria está formada por 10 bandas beta hidrofóbicas, las cuales semejan un cilindro semi-aplanado abierto en uno de sus extremos y cerrado en el extremo opuesto por 2 hélices alfa. En el interior del cilindro, las unidades beta son el sitio de unión específico del AG de cadena larga, capaz de unir una molécula del ligando a través de la interacción de fuerzas hidrofóbicas y electroestáticas. La afinidad con el ligando depende de la afinidad del AG a las cadenas laterales de las unidades beta de la proteína 5 .

Aunque su papel fisiológico aún no está perfectamente aclarado, las hipótesis sobre las funciones de las FABPs incluyen la neutralización de los AG en el citosol, minimizando sus efectos tóxicos en la célula al evitar interacciones con membranas o solutos celulares. Además, regularían la síntesis y secreción lipídica en las células intestinales, probablemente influyendo sobre el transporte intracelular de AG hacia organelos celulares. Por otro lado, participarían en la modulación génica a través de señales de transducción lipídicas. Las FABPs, como transportadores celulares, transportan hacia el núcleo lípidos regulatorios, influyendo en la expresión génica de PPAR ${ }^{5,9,10}$. Los AG poliinsaturados de cadena larga actúan como ligandos del PPAR 2 y otros receptores de la familia de los PPAR; estas uniones son capaces de inducir o reducir la diferenciación de los adipocitos 9,11 . Finalmente, la absorción diferencial de AG puede influir en la composición fosfolipídica de la membrana celular contribuyendo al estado de leve inflamación crónica que acompañan a la RI y patologías derivadas.

Estudios efectuados in vitro, con células humanas de carcinoma de colon (Caco2), han demostrado que la variante Thr54 tiene el doble de afinidad por ácidos grasos de cadena larga que la forma nativa y que secreta ésteres de colesterol y TG en forma más eficiente que la forma Ala54Ala ${ }^{12}$. Otros estudios efectuados in vitro, en células yeyunales de intestino humano, heterocigotos para la variación Ala54Thr, mostraron que la síntesis y secreción de triglicéridos fue significativamente mayor en ellas comparadas con la variante nativa ${ }^{13}$. El polimorfismo de FABP2 más frecuente y extensamente estudiado es la sustitu- 
ción de alanina (Ala) por treonina (Thr) en el gen de un nucleótido en el codón 54 del exón 2. El producto de esta variante del gen, la variante Ala54Thr de la FABP2, tiene alterada la función y ha sido asociado con RI y alteración de la oxidación de los ácidos grasos ${ }^{14}$.

\section{Prevalencia}

Los polimorfismos genéticos de FABP2 se han estudiado extensamente en cuanto a su asociación con obesidad, diabetes tipo 2 y $\mathrm{RI}^{5}$. La Tabla 1 ilustra la frecuencia alélica de FABP2 en estudios publicados para definir el papel y la frecuencia de la variación Ala54Th. Se trata de una mutación relativamente frecuente, con frecuencias alélicas de 30 a $40 \%$ en las poblaciones estudiadas ${ }^{1}$. En indios Pima se encontró una frecuencia de $0,31^{14}$, en coreanos $0,34^{15}$, en japoneses $0,35^{16}$, en suecos $0,30^{17}$, en franco-canadienses $0,31^{10}$, en aborígenes canadienses $0,35 \% 18$, en mujeres obesas chilenas $0,40^{19}$, en ancianos chilenos $0,39^{20}$, en mapuches $0,32^{21}$ y en aymaras $0,18 \% \%^{21}$. La distribución de los genotipos es similar en los diferentes grupos estudiados, con la excepción del grupo de aymaras estudiado por Pérez y cols $^{21}$, en los cuales se encontró la más baja frecuencia alélica descrita hasta la fecha.

\section{Polimorfismo De FABP2 y pRoblemas metabólicos}

Las manifestaciones fenotípicas sobre el metabolismo lipidíco y glucídico, asociadas a la variante 54Thr, no son comparables ni concluyentes en las diferentes publicaciones.

Ello, probablemente, se debe a que las metodologías utilizadas para determinar RI difieren entre un estudio y otro -siendo el $\operatorname{HOMA}^{22}$ y la insulinemia los más utilizados- o bien a tamaños muestrales insuficientes ${ }^{5,23,24}$. Por otra parte, la interacción genético/ambiental es de especial relevancia en la etiopatogenia de la RI y la diabetes tipo 2. La dieta, la falta de actividad física, la composición corporal, la obesidad y el sobrepeso

Tabla 1. G enotipo FABP2 y frecuencia alélica en algunas poblaciones estudiadas

\begin{tabular}{|lcccccc|}
\hline Grupo de estudio & Ala/Ala & Ala/Thr & Thr/Thr & Ala & Thr & Ref. \\
\hline $\begin{array}{l}\text { Indios Pima } \\
\text { (n=457) }\end{array}$ & 0,48 & 0,45 & 0,07 & 0,70 & 0,30 & 14 \\
$\begin{array}{l}\text { Japoneses } \\
\text { (n=297) }\end{array}$ & 0,41 & 0,49 & 0,11 & 0,65 & 0,35 & 26 \\
$\begin{array}{l}\text { Coreanos } \\
\text { (n=96) }\end{array}$ & 0,45 & 0,42 & 0,13 & 0,66 & 0,34 & 15 \\
$\begin{array}{l}\text { Chinos } \\
n=165\end{array}$ & 0,45 & 0,44 & 0,11 & 0,67 & 0,33 & 27 \\
$\begin{array}{l}\text { Suecos } \\
\text { (n=59) }\end{array}$ & 0,42 & 0,46 & 0,12 & 0,70 & 0,30 & 17 \\
$\begin{array}{l}\text { Finlandeses } \\
n=140\end{array}$ & 0,49 & 0,45 & 0,06 & 0,72 & 0,28 & 29 \\
$\begin{array}{l}\text { Mujeres USA } \\
\text { (n=60) }\end{array}$ & 0,61 & 0,30 & 0,09 & 0,76 & 0,24 & 23 \\
$\begin{array}{l}\text { Mujeres Chile } \\
\text { (n=63) }\end{array}$ & 0,37 & 0,47 & 0,16 & 0,60 & 0,40 & 19 \\
$\begin{array}{l}\text { Adultos mayores } \\
\text { chilenos (n=813) }\end{array}$ & 0,36 & 0,43 & 0,21 & 0,61 & 0,39 & 20 \\
$\begin{array}{l}\text { Mapuches (Chile) } \\
\text { Aymaras (Chile) }\end{array}$ & 0,66 & 0,31 & 0,03 & 0,68 & 0,32 & 21 \\
\hline
\end{tabular}


son factores de riesgo independientes para diabetes y RI. Ello hace muy difícil la comparación de estudios cuyos resultados podrían estar sesgados si estos factores confundentes no se han considerado en el diseño y análisis.

En la Tabla 2 se describen los resultados de estudios en búsqueda de asociación del polimorfismo Thr54 con RI y otras alteraciones metabólicas en diferentes etnias y razas.

En indios Pima no diabéticos se evidenció asociación del polimorfismo Thr54 con insulino- rresistencia, aumento de la concentración de insulina y de la oxidación de ácidos grasos en ayunas, pero sin cambios en el peso corporal ${ }^{14}$. En mexicanos-americanos se encontró asociación entre este polimorfismo y aumento de la insulinemia de $2 \mathrm{~h}$ postcarga de glucosa ${ }^{25}$. En población aborigen canadiense, se encontró aumento del IMC y TG en ayunas en portadores del alelo Thr $54^{18}$

En hombres coreanos sanos, el polimorfismo Thr54 de la FABP2 se asoció con aumento de la

\section{Tabla 2. Estudios en búsqueda de asociación del polimorfismo Ala54T hr de la FABP2 con RI y alteraciones metabólicas relacionadas}

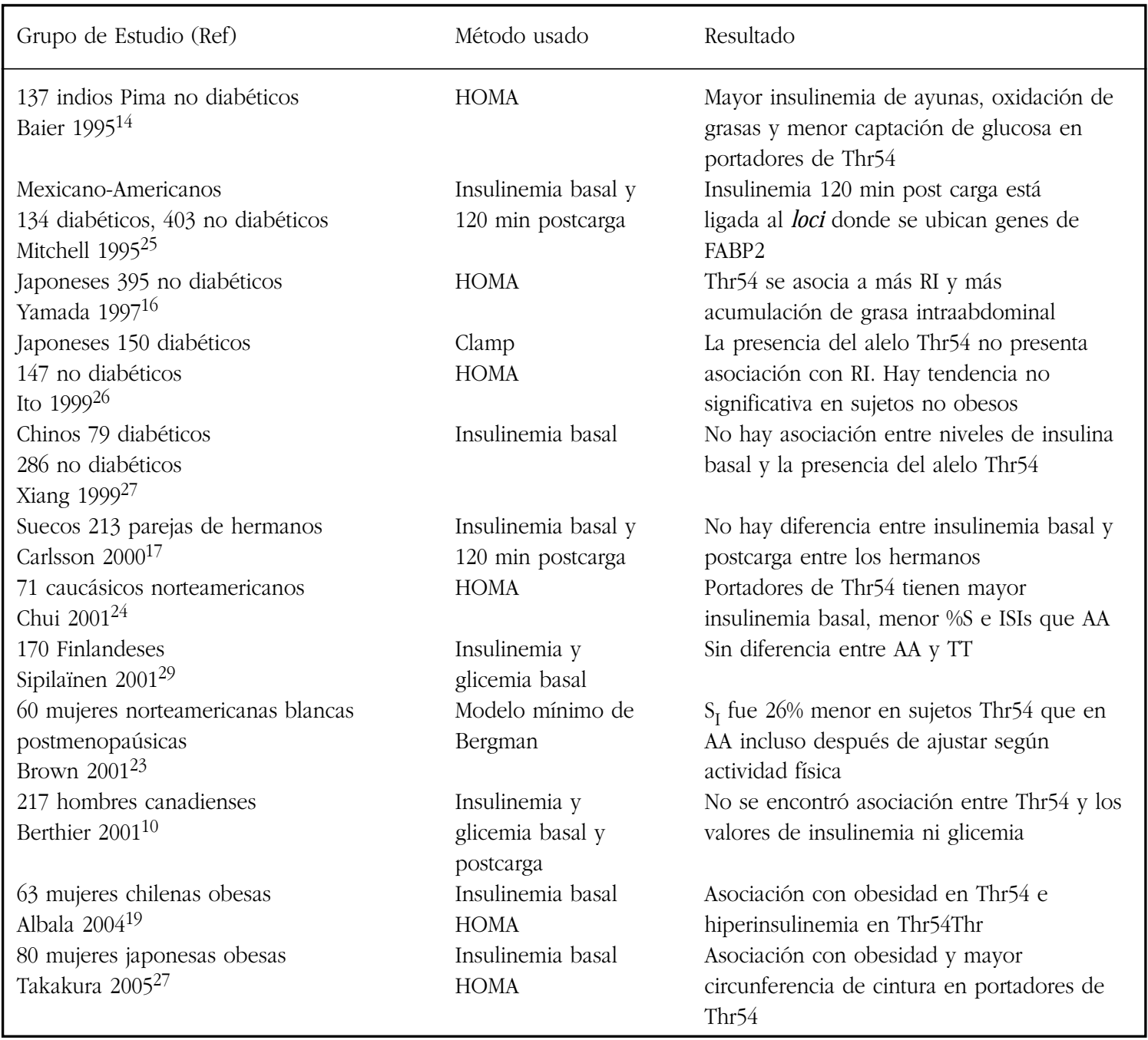


oxidación de AG y de niveles de insulinemia basal, aunque no se observó incremento en la absorción de los $\mathrm{AG}^{15}$. En estudios efectuados en hombres japoneses, por Yamada y cols ${ }^{16}$, el alelo Thr 54 mostró asociación con dislipidemia e insulinorresistencia y aumento de la grasa abdominal en homocigotos para Thr54. Sin embargo, estudios efectuados por Ito y cols, en $1999^{26}$, en japoneses diabéticos y no diabéticos no encontraron asociación con diabetes. Recientemente, Takakura y $\mathrm{cols}^{27}$, han repetido un resultado similar en mujeres japonesas obesas, donde se observa asociación entre el alelo Thr54 con obesidad y mayor diámetro de cintura. Otros estudios en asiáticos, como los realizados por Xiang y cols en $1999^{28}$, en chinos diabéticos y no diabéticos, solo demostraron disminución de grasa femoral en portadores del alelo Thr54 en diabéticos.

Estudios en población afroamericana, como los de Lei y $\operatorname{cols}^{29}$, no pudieron demostrar ninguna asociación entre Thr54 e hiperinsilunemia, diabetes u obesidad.

Las investigaciones realizadas en poblaciones de origen caucásico también han sido contradictorias. En un estudio en sujetos caucásicos americanos sanos, se encontró que el polimorfismo Ala54Thr de FABP2 se asoció con RI y disminución de la sensibilidad insulínica en comparación con el alelo nativo, pero sin disfunción de las células beta del páncreas ${ }^{23}$. El estudio de Albala y cols $^{19}$, realizado en 63 mujeres chilenas premenopáusicas obesas, encontró asociación del alelo Thr54 con obesidad, además de insulina plasmática de ayuno elevada en homocigotos Thr54Thr. Sin embargo, estudios realizados en poblaciones europeas no han encontrado asociación con $\mathrm{RI}^{23}$. Asimismo, en franco-canadienses obesos la mutación no contribuyó significativamente a la obesidad ni al hiperinsulinismo ${ }^{10}$.

Desde el punto de vista del metabolismo lipídico, las asociaciones encontradas también son contradictorias: mientras en población caucásica de Finlandia $^{30}$ y en escolares japoneses ${ }^{31}$ la variante genética no ha sido asociada a obesidad o alteración en el metabolismo lipídico o glucídico, en otros estudios ${ }^{32}$ se ha observado asociación entre el alelo Thr54 y colesterol LDL bajo. No obstante, la asociación entre Thr54 y perfil lipídico es incluso contradictoria en estudios en familias con hiperlipidemia e hipercolesterolemia ${ }^{33,34}$.
Publicaciones recientes han examinado el rol del polimorfismo Ala54Thr FABP2 sobre algunos parámetros del síndrome metabólico, encontrándose una modesta alza en la presión arterial diastólica y en el colesterol LDL para los homocigotos Thr54Thr; también este genotipo se asoció a un leve incremento máximo del grosor de la capa íntima media de las carótidas en hombres 35,36 .

Según las hipótesis postuladas por Baier y cols $^{14}$, la asociación del polimorfismo a RI y diabetes tipo 2 se explicaría por una hipertrofia de los adipocitos producido por el aumento de la absorción de grasa dietaria ${ }^{18,23}$, asociado a un balance calórico positivo. En este adipocito hipertrófico se produciría la disminución subsecuente de la densidad de receptores de insulina en su membrana, lo cual llevaría a RI de las células adiposas, alterando la glicólisis, disminuyendo la esterificación de ácidos grasos y permitiendo que ellos pasen al torrente sanguíneo. El incremento de los ácidos grasos circulantes aumenta la gluconeogénesis hepática, cuyo producto es almacenado como glicógeno o liberado a la sangre. También se podría producir un acúmulo lipídico en el miocito, aumentando la insulinorresistencia y el hiperinsulinismo compensatorio, lo que finalmente llevaría a disfunción de las células beta pancreáticas y a la elevación de la glucosa plasmática.

Nuestros estudios sugieren que la presencia del polimorfismo A54T se asocia en forma independiente de la obesidad con un aumento de citoquinas inflamatorias contribuyendo de esta manera al leve estado inflamatorio crónico que se observa en la obesidad y que se asocia a RI ${ }^{19}$. Ello podría explicarse por absorción diferencial de los ácidos grasos polinsaturados $\mathrm{n}-6$, aumentando el valor de la relación n- $6 / \mathrm{n}-3$, los cuales provocan mayor respuesta de citoquinas inflamatorias asociadas a disminución de la sensibilidad a la insulina ${ }^{37}$.

Otro aspecto que también ha sido evaluado en relación a la presencia del polimorfismo $54 \mathrm{~T}$ es la absorción diferencial de AG. Un estudio efectuado en ratas por Richieri et al en $1994^{38}$, demostró mayor afinidad de FABP2 por ácido palmítico, sugiriendo que la afinidad de la proteína que contiene Thr cambia según el tipo de ácido graso ingerido. Agreen y cols ${ }^{39}$, relacionan la presencia del alelo Thr54 con la absorción diferencial de 
ácidos grasos ingeridos, encontrando un incremento significativo en quilomicrones y VLDL posterior a la carga de AG de carbonos 14-18 en comparación a homocigotos Ala. Asimismo, en sujetos homocigotos para el alelo Thr, se ha encontrado una mayor trigliceridemia asociada a una respuesta alterada de insulina post-prandial. Recientemente, el estudio de la composición de quilomicrones postcarga oral de diferentes ácidos grasos, mostró absorción diferencial en portadores de la mutación Thr54 sólo para ácido oleico, no así para ácidos grasos de cadena corta o media$\mathrm{na}^{40}$. Sin embargo, a pesar de que estos resultados resultan muy llamativos desde la perspectiva de la posible interacción dieta-genotipo, algunos estudios previos diseñados en modelos animales transgénicos para este mutante no mostraron igual asociación con la absorción de lípidos ${ }^{41}$. Por otra parte, investigaciones recientes sugieren la asociación de Ala54Thr, con una variante ligada al promotor de FABP2 que alteraría su expresión, de tal forma que en algunos casos este último polimorfismo podría ser el responsable del fenotipo ligado a variantes de FABP2 ${ }^{42}$.

\section{RESUMEN Y CONCLUSIONES}

La obesidad, la RI y la diabetes tipo 2 corresponden a entidades complejas tanto desde el punto de vista genético, como metabólico. La gran cantidad de genes involucrados, así como su heterogeneidad, hacen muy compleja su evaluación. La variación interindividual en la respuesta metabólica a dietas y ejercicio, hace que la evaluación de determinados genes candidatos,

\section{REFERENCIAS}

1. Mercado MM, McLenithan JC, Silver KD, Shuldiner AR. Genetic of Insulin Resistance. Curr Diab Rep 2002; 2: 83-95.

2. Damcott CM, Sack P, Shuldinger AR. The genetics of obesity. Endocrinol Metab Clin NAm 2003; 32: 761-86.

3. Elbein SC, Maxwell TM, Schumacher MC. Insulin and glucose levels and prevalence of glucose que tienen variantes comunes funcionales en la población general, puedan ser determinantes en las diferencias interindividuales de la respuesta a los componentes de la dieta, como la grasa dietética y la fibra.

La FABP2 es una proteína intracelular expresada sólo en el intestino delgado e involucrada en la absorción y transporte intracelular de ácidos grasos de cadena larga. Los portadores del alelo Thr54 en FABP2 tienen el doble de afinidad por ácidos grasos de cadena larga que aquellos con la forma nativa de la FABP2. El aumento del flujo de ácidos grasos dietarios en la circulación puede llevar a una alteración de la sensibilidad del metabolismo de la glucosa a la insulina, lo que avalaría el papel de la variante Ala54Thr de la FABP2 en la etiología de desórdenes metabólicos.

En muchos grupos poblacionales se ha investigado una asociación entre polimorfismo de FABP2 y trastornos metabólicos y, aunque los resultados no son concluyentes, en general se ha observado relación con RI o sus consecuencias metabólicas. Sin embargo, considerando que los trastornos metabólicos mencionados (RI, alteraciones del metabolismo de la glucosa, obesidad, dislipidemia) son condiciones que se han ligado a varios genes candidatos, la evidencia de asociaciones causales para FABP2 ligadas a respuestas adaptativas en enfermedades complejas como las descritas, debe ser analizada en el contexto de la interacción entre múltiples genes, donde la epistasis genética y la interacción gen-ambiente son cruciales $33,36,43,44$. Es por ello que, en la práctica actual, la determinación del polimorfismo Ala54Thr de FABP2 no tiene aplicación para establecer el riesgo de obesidad e insulinorresistencia en la población.

intolerance in pedigrees with multiple diabetic siblings. Diabetes 1991; 40: 1024-32.

4. Mayer EJ, Newman B, Austin MA, Quesenberry CP, Edwards K, Selby JB. Genetic and environmental influences on insulin levels and the insulin resistance syndrome: an analysis of women twins. Am J Epidemiol 1996; 146: 323-32.

5. Waiss EP, Brown MD, Shuldiner AR, Hagberg JM. Fatty acid binding protein-2 gene variants and insulin resistance: gene and gene- environment 
interactions effects. Physiol Genomics 2002; 10 : 145-57.

6. Bernlohr DA, Simpson MA, VogelHertzel A, BanasZAK LJ. Intracellular lipid-binding proteins and their genes. Ann Rev Nutr 1997; 17: 277-303.

7. Hsu KT, Sтоrсh J. Fatty acid transfer from liver and intestinal fatty acid binding proteins to membranes occurs by different mechanisms. J Biol Chem 1996; 271: 13317-23.

8. Sparkes RS, Mohandas T, Heinzmann C, Gordon JI, KLISAK I, Zollman S et al. Human fatty acid binding protein assignments: intestinal to $4 \mathrm{q} 28-4 \mathrm{q} 31$ and liver to 2p11. (Abstract) Cytogenet Cell Genet 1987; 46: 697.

9. ORdovas JM. Genetics, postprandial lipemia and obesity. Nutr Metab Cardiovasc Dis 2001; 11: 118-33.

10. Berthier MT, Coulllard C, Prudhomme D, Nadeau A, Bergeron J, Tremblay A et al. Effects of the FABP2 A54T mutation on triglyceride metabolism of viscerally obese men. Obes Res 2001; 9: 668-75.

11. LeWIS MR, TRacy RP. The role of the immune system in the insulin resistance syndrome. Curr Diab Rep 2002; 2: 96-9.

12. Baier LJ, Bogardus C, Sacchettini JC. A Polymorphism in the human intestinal fatty acid binding protein alters fatty acid transport across Caco 2 cells. J Biol Chem 1996; 271: 10892-6.

13. Levy E, Menard D, Delvin E, Stant S, Mitchell G, LAMBERT M ET AL. The polymorphism at codon 54 of the FABP2 gene increases fat absorption in human intestinal explants. J Biol Chem 2001; 276: 39679-84

14. Baier LJ, Sacchettini JC, Knowler WC, Eads J, Paolisso G, Tataranni Pa et al. An amino acid substitution in the human intestinal fatty acid binding protein is associated with increased fatty acid binding, increased fat oxidation and insulin resistance. J Clin Invest 1995; 95: 1281-7.

15. Kim Ch H, Yun SK, Byun DW, Yoo MH, Lee KU, SuH KL. Codon 54 polymorphism of the FABP2 gene is associated with increased fat oxidation and hyperinsulinemia but not with intestinal fatty acid absorption in Korean men. Metabolism 2001; 50: 473-6.

16. Yamada K, Yuan X, Ishiyama $S$, Koyama $\mathrm{K}$, Ichikawa F, Koyanagi A et al. Association between Ala54Thr substitution on the fatty binding protein 2 gene with insulin resistance and intra-abdominal fat thickness in Japanese men. Diabetologia 1997; 40: $706-10$.
17. Carlsson M, Orho-Melander M, Hedenbro J, AlMgren P, GRoOp LC. The T 54 allele of the intestinal fatty acid binding protein 2 is associated with parental story of stroke. J Clin Endocrinol Metab 2000; 85: 2801-4.

18. Hegele RA, Connelly PW, Hanley AJ, Sun F, Harris $\mathrm{SB}$, Zinman B. Common genomic variants associated with variations in plasma lipoproteins in young aboriginal Canadians. Arterioscler Thromb Vasc Biol 1997; 17: 1060-6.

19. Albala C, Santos Jl, Cifuentes M, Villarroel AC, Lera L, Liberman C et al. Intestinal FABP2 A54T polymorphism: Association with insulin resistance and obesity in women. Obes Res 2004; 12: 3405.

20. Albala C, Angel B, Lera L, Cifuentes M, Villarroel AC, SAntos JL ET AL. Variants in the human Intestinal Fatty Acid Binding protein 2 gene in Chilean elders. Obes Res 2003, 11(supp) 47.

21. Pérez-Bravo F, Fuentes M, Angel B, Cifuentes M, Carrasco E, Santos J et al. Lack of Association Between the Fatty Acid Binding Protein 2 (Fabp-2) Polymorphism With Obesity and Insulin-Resistance in two Aboriginal Populations from Chile. Eur J Med Genet 2006, en prensa.

22. MatThews DR, Hosker JP, Rudenski AS, NAYlor BA, Treacher DF, Turner RC. Homeostasis model assessment: insulin resistance and b-cell function from fasting plasma glucose and insulin concentrations. Diabetologia 1985; 28: 412-18.

23. Brown M, Shuldiner A, Ferrell R, Weiss E, KorytKowski Zmuda J, McCole S et al. FABP2 genotype is associated with insulin sensitivity in older women. Metabolism 2001; 50: 1102-5.

24. Chui KC, Chuan L-M, Yoon C. The A54T polymorphism at the intestinal fatty acid binding protein 2 is associated with insulin resistance in glucose tolerant Caucasians. BMC Genetics 2001; 2: 7-15.

25. Mitchell B, Kammerer C, O'Connell C, Harrison Ch, Manire M, Shipmen P et al. Evidence for linkage of postchallenge insulin levels with intestinal fatty acid binding protein (FABP2) in Mexican-Americans. Diabetes 1995; 44: 1046-53.

26. Ito K, Nakatani K, Fuji M, Katsuki A, Tsuchinashi K, Murata K ET AL. Codon 54 polymorphism of the fatty acid binding protein gene and insulin resistance in the Japanese population. Diabetic Medicine 1999; 16: 119-24.

27. Takakura Y, Yoshioka K, Umekawa T, Kogure A, 
Toda H, YoshiKawa T ET al. Thr54 allele of the FABP2 gene affects resting metabolic rate and visceral obesity. Diab Res Clin Pract 2005; 67: 3642.

28. Xiang K, Zhengt, Jia W, Sun D, Ding W, Li J et al. The association of Ala54Thr variant of intestinal fatty acid binding protein gene with general and regional adipose tissue depots. Chin Med Sci J 1999; 14: 46-51.

29. Lei H, Coresh J, Schuldiner A, Boerwinkle E, BREANCATI F. Variants of the insulin receptor substrate- 1 and fatty acid binding protein 2 genes and the risk of type 2 diabetes, obesity and hyperinsulinemia in African-Americans. Diabetes 1999; 48: 1868-72.

30. Sipilaïnen R, Uusitupa M, Heikkinen S, Rissanen A, LAAKSO M. Variants in the Human fatty acid binding protein 2 gene in obese subjects. J Clin Endocrinol Metab 1997; 82: 2629-32.

31. Endo K, Yanagi H, Hirano C, Hayakawa Y, HamaguSHI H, Tomura S. No association found between the Ala54Thr polymorphism of FABP2 gene and obesity and obesity with dyslipidemia in Japanese school children. J Atheroscler Thromb 2001; 8: 80-3.

32. Duarte NL, Colagiuri S, Palu T, Wang XL, Wilcken DE. Obesity, type II diabetes and the Ala54Thr polymorphism of fatty acid binding protein 2 in the Tongan population. Mol Genet Metab 2003; 79: $183-8$.

33. Campagna F, Montali A, Baroni G, Maria AT, Ricci G, Antonini R et al. Common variants in the lipoprotein lipase gene, but not those in the insulin substrate-1, the beta3-adrenergic receptor and the intestinal fatty acid binding protein-2 genes, influence the lipid phenotypic expression in familial combined hyperlipidemia. Metabolism 2002; 51: 1298-305.

34. Bertolini S, Pisciotta L, Di Scala L, Langheim S, Bellocchio A, Masturzo P et al. Genetic polymorphism affecting the phenotypic expression of familial hypercholesterolemia. Atherosclerosis 2004; 174: 57-65.

35. Renner W, Pressl H, Wascher TC, Paulweber B, IGLSEDER B. The role of the A54T polymorphism of the intestinal fatty acid binding protein for lipid levels, insulin sensitivity and carotid atherosclerosis. Atherosclerosis 2004; 173: 137-9.

36. Guettier JM, Georgopoulos A, Tsai MY, Radha V,
Shanthirani S, Deppa R et al. Polymorphism in the fatty acid-binding protein 2 and apolipoprotein C-III genes are associated with the metabolic syndrome and dyslipidemia in South Indian population. J Clin Endocrinol Metab 2005; 90: 1705-11.

37. Endres S, Ghorbani R, Kelley VE, Georgilis K, Lonnemann G, van der MeER JW ET aL. The effect of dietary supplementation with $\mathrm{n}-3$ polyunsaturated fatty acids on the synthesis of interleukin-1 and tumor necrosis factor by mononuclear cells. N Engl J Med 1989; 320: 256-71.

38. Richieri GV, Ogata RT, Kleinfeld AM. Equilibrium constants for the binding of fatty acids with fatty acid-binding proteins from adipocyte, intestine, heart, and liver measured with the fluorescent probe ADIFAB. J Biol Chem 1994; 269: 23918-30.

39. Agren J, Vidgren H, Valve R, Laakso M, Uusitupa M. Postprandial responses of individual fatty acids in subjects homozygous for the threonine or alanine-encoding allele in codon 54 of the intestinal fatty acid binding protein 2 gene. Am J Clin Nutr 2001; 73: 31-5.

40. Dworatzek P, Hegele R, Wolever T. Postprandial lipemia in subjects with the threonine 54 variant of the fatty acid binding protein 2 gene is dependent on the type of fat ingested. Am J Nutr 2004; 79: 1110-17.

41. Vassileva G, Huwyler L, Poirier K, Agellon LB, Тотн MJ. The intestinal fatty acid binding protein is not essential for dietary fat absortion in mice. FASEB J 2000; 14: 2040-6.

42. Formanack ML, Baier LJ. Variation in the FABP2 promoter affects gene expression: implications for prior association studies. Diabetologia 2004; 47: 349-51.

43. Yanagisawa Y, Kawabata T, Tanaka O, Kawakami M, Hasegawa K, Kagawa Y. Improvement in blood lipid levels by dietary sn-1,3-diacylglycerol in young women with variants of lipid transporters 54T-FABP2 and -493-MTP. Biochem Biophys Res Commun 2003; 302: 743-50.

44. Ribalta J, Halkes CJ, Salazar J, Masana L, Cabezas MC. Additive effects of the PPAR gamma, APOE and FABP-2 genes in increasing daylong triglycerides of normolipidemic women to concentrations comparable to those in men. Clin Chem 2005; 51: 864-71. 